\title{
Geographically weighted methods for estimating local surfaces of overall, user and producer accuracies
}

\author{
ALEXIS J. COMBER \\ Department of Geography \\ University of Leicester \\ Leicester \\ LE1 7RH \\ UK \\ Email: ajc36@le.ac.uk
}

\begin{abstract}
The confusion matrix is the standard way for reporting the accuracy of land cover and other information classified from remote sensing imagery. This letter describes a geographically weighted method for generating spatially distributed measures of accuracy (overall, user and producer accuracies) from a logistic Geographically Weighted Regression. A kernel-based approach defines the data and weights that are used to calculate the accuracies at each location in the study area. The results compare the global accuracy measures from a standard confusion matrix with ones that have been allowed to vary locally. Maps of spatially varying user and producer accuracies describe the spatial autocorrelation of error. The use of geographically weighted models in the context of land cover accuracy are discussed and suggested as a generic approach for examining how and where error processes vary.
\end{abstract}

\section{Introduction}

The confusion matrix and the measures it supports such as user accuracy and producer accuracy have become the accepted paradigm for reporting accuracy in remote sensing and land cover. However, the information provided by the confusion matrix is aspatial it does not tell potential users where the data are better or worse and does not describe the spatial distribution of errors (Canters 1997; Steele et al., 1998; McGuire and Fisher, 2001; Foody, 2002; Foody, 2005; Foody, 2007). The tendency for errors in land cover data to be spatially autocorrelated is well known (from Campbell 1981 to Gonzalez et al., 2010). To overcome this, some research has developed methods for estimating and describing the spatial distribution land cover accuracy. Of particular relevance to the methods proposed here are the work of Foody (2005) and Comber et al. (in press). Foody developed geographically distributed measures of accuracy, calculating confusion matrices at a series of locations and then interpolating between them to create surfaces of error. Comber et al. extended Foody's work and proposed a geographically weighted measure of portmanteau accuracy for Boolean land cover and a geographically weighted difference measures for fuzzy land cover.

This letter outlines geographically weighted methods for generating spatially varying measures of land cover accuracies. Geographically weighted approaches use a kernel or moving window to make local calculations using the data points that are under the kernel, but whose contribution to the calculation is weighted by their distance to the 
kernel centre. The methods extend Foody (2005) by incorporating distance weighting and extend Comber et al. (in press) by developing geographically weighted estimates of the spatial distribution of user, producer and overall accuracies.

\section{Methods}

\subsection{Data}

SPOT 5 imagery from 2009 for area in the North Western part of Libya around Tripoli was resampled to $30 \mathrm{~m} \times 30 \mathrm{~m}$ as part of a wider study. It was classified into 6 classes using the maximum likelihood classifier in the Idrisi Maxlike module: Urban, Woodland, Vegetation, Grazing Land and Bare. A field survey in 2010 collected validation data at 210 locations selected by stratified random sample that divided the study areas into blocks, from which an average of 10 locations per block were selected randomly. The dataset used in the analysis contained data from the field survey ("reference" data) and from the remote sensing analysis ("classified data") at each of the 210 locations. The study area and the locations of the sample points are shown in Figure 1 along with the grid used in the geographically weighted analyses (described below). Table 1 shows the confusion matrix arising from the validation. Overall accuracy is calculated from the diagonal and off diagonal elements in the confusion matrix. User and producer accuracies are calculated using the row and column totals. Overall accuracy describes the proportion of the total number of pixels that have the same class in the reference and classified data for all classes. User and producer accuracies describe the errors related to individual classes.

(insert Figure 1 about here)

(insert Table 1 about here)

\subsection{Aspatial Analysis}

It is informative to examine how the probabilities related to these accuracy measures can also be estimated using ordinary logistic regressions. First, a logit function needs to be defined to transform any value, $q$ :

$$
\operatorname{logit}(q)=\frac{\exp (q)}{1+\exp (q)}
$$

Overall accuracy, $A_{O}$, can be estimated using a reduced logistic regression model that only contains an intercept term, $b$ (i.e. it seeks to estimate the overall accuracy using an explanatory variable of 1$)$ :

$A_{O}=\operatorname{logit}(b)$

(Equation 2)

which returns an estimate of the probability of $A_{O}$ being equal to 1. When Equation 2 is used to analyse the confusion matrix data in binary form, where the 126 data points summarised by the diagonal elements are indicated as 1 and the 84 data points 
summarised by the off-diagonal elements as 0 , the result is an estimate of the overall accuracy of 0.60 .

Now consider a subset of the data for the class of Grazing Land. It is represented by two similarly ordered binary vectors of 210 elements, with the reference data vector having 39 elements indicated by 1 and the classified data vector having 43 . User accuracy can be estimated using a logistic regression to analyse the reference data against the classified data in the following way:

$P(y=1)=\operatorname{logit}\left(b_{0}+b_{1} x_{1}\right)$

(Equation 3)

where $P(y=1)$ is the probability that the reference land cover class, $y$, is correctly predicted at by the classified data, $x_{1}, b_{0}$ is the intercept term and $b_{1}$ is the slope. The result describes the probability that the reference data equals 1 , given that the classified data equals 1 (i.e. the user accuracy), which in this case is equal to 0.53.

Inverting the response and explanatory variables in Equation 3 estimates producer accuracy:

$P(x=1)=\operatorname{logit}\left(b_{0}+b_{1} y_{1}\right)$

(Equation 4)

where $P(x=1)$ is the probability that the classified land cover class is correctly predicted by the reference data, $y_{1}, b_{0}$ is the intercept term and $b_{1}$ is the slope. Using the ordered binary vectors described above, the producer accuracy for Grazing Land is equal to 0.59 .

\subsection{Geographically weighted analysis}

Having outlined the probabilities related to the principal accuracies arising from the confusion matrix in terms of logistic regression, it is now possible to consider the geographically weighted extensions to these models. They are similar in form to ordinary regression but geographically weighted approaches use a moving window or kernel under which local models such as a logistic regression are computed at locations through the study area, in this case the $1 \mathrm{~km}$ grid shown in Figure 1.

The aspatial models described in Equations 2, 3, and 4 were extended to geographically weighted models in the following way:

$$
\begin{aligned}
& P\left(A_{O}=1\right)=\operatorname{logit}\left(b_{O\left(u_{i}, v_{i}\right)}\right) \\
& P\left(y_{i}=1\right)=\operatorname{logit}\left(b_{O\left(u_{i}, v_{i}\right)}+b_{I} x_{I\left(u_{i}, v_{i}\right)}\right) \\
& P\left(x_{i}=1\right)=\operatorname{logit}\left(b_{O\left(u_{i}, v_{i}\right)}+b_{I} y_{I\left(u_{i}, v_{i}\right)}\right)
\end{aligned}
$$


where $\operatorname{pr}\left(A_{O}=1\right)$ is the overall accuracy probability at location $i, x_{1}$ and $y_{1}$ are the classified and reference data respectively and $\left(u_{i}, v_{i}\right)$ is a vector of two dimensional coordinates describing the location of $i$ over which the coefficient estimates are assumed to vary.

In contrast to ordinary regression models where the processes under investigation are assumed to be stationary (i.e. location invariant), local models are spatial

disaggregations of global models, the results of which are location-specific. A moving window allows a local regression to be calculated at each location and data points that are further away from the location under consideration contribute less to the solution. Thus the kernel defines the data and weights that are used to calibrate the model at each location. The weight, $w_{i}$, associated with each location $\left(u_{i}, v_{i}\right)$ is a decreasing function of $d_{i}$, the distance from the centre of the kernel to $\left(u_{i}, v_{i}\right)$ :

$$
w_{i}=\left\{\begin{array}{cc}
\left(1-\frac{d_{i}^{2}}{h^{2}}\right)^{2} & \text { if } d_{i}<h \\
0 & \text { otherwise }
\end{array}\right.
$$

\section{(Equation 8)}

where $h$ is the bandwidth and the weights for each data point, $i$, change for each location. Here, the bandwidth was selected to include the nearest $15 \%$ of the data points.

\section{Results}

The geographically weighted logistic regressions described in Equations 5, 6 and 7 were applied to the land cover class of Grazing Land over the $1 \mathrm{~km}$ grid illustrated in Figure 1. Table 2 summarises the distribution of overall accuracy and user and producer accuracies for Grazing Land, as calculated at each grid cell. Overall accuracy varies from 0.57 to 0.65 in different parts of the study area compared to a global value of 0.60 . User accuracy varies from 0.13 to 0.71 , compared to a global figure of 0.53 and producer accuracy for varies from 0.52 to 0.72 across the study area, compared to a global figure of 0.59 . It is interesting to note that there are much greater variation in the distribution of user accuracy estimates, moderate variation in producer accuracy estimates and less variation in the estimates of overall accuracy.

(insert Table 2 about here)

The spatial variations in user and producer accuracies are shown in Figures 2 and 3. Figure 2 indicate that the user accuracy for Grazing Land is generally higher to the south of the study area, and lower towards the north (around Tripoli). User accuracy provides an estimate of the probability that a classified pixel correctly represents the features on the ground. It indicates of the errors of commission (inclusion) and for the potential user of the map it indicates the probability of correctly finding that class on the ground. In this case the results in Figure 2 would suggest less confidence in the areas mapped as Grazing Land around Tripoli being that class on the ground. Figure 3 shows that there is much less variation in the estimates of Grazing Land producer accuracy, but that there is a trend of marginally higher accuracy towards the north of the study area. 
Producer accuracy provides an estimate of the probability that a reference pixel is correctly labeled in the classified data. It indicates the errors of omission (exclusion) and for the producer of the map it indicates the probability that features of interest are omitted from the classified data. Thus, in this case, we can be confident that most of the grazing land that exists has been mapped (low omission) but that there are also high levels of commission errors in the north of the study area.

(insert Figure 2 about here)

(insert Figure 3 about here)

\section{Discussion}

The confusion matrix provides a convenient summary of land cover errors but it provides only global measures of error and accuracy and is aspatial in nature. However, descriptions of the local spatial variation in land cover accuracy are useful as land cover (and land use) data are used as inputs to many environmental and socio-economic models. Determining the local variations in accuracy can help users assess the suitability of land cover data for their application and assess the implications of those errors for their analyses. Geographical analyses are fundamentally concerned with how and why processes vary spatially. They explicitly use the locational attributes of data to identify local variations in relationships. This is not the same as analysing spatial data per se and assuming that because the data is spatial, then its analysis somehow represents a 'spatial analysis'. This sometimes appears to be the case in the remote sensing community where the failure to adopt spatially explicit approaches is puzzling, especially as it is well recognised that phenomena such as land cover errors cluster for a variety of well documented reasons, and that there is a need for local error assessments which are often by informal visual and qualitative methods (Freidl et al., 2002).

This letter shows how logistic regression can be used to generate probabilities such as user and producer accuracies and that geographically weighted extensions are able to generate spatial distributions that describe the variation of these probabilities. Other research has suggested geographically weighted approaches and this research is very much in the spirit of Foody (2005) and Comber (in press). Foody (2005) developed a method for describing the geographical distribution of error. Comber et al. (in press) proposed and developed a geographically weighted portmanteau measure of accuracy. Portmanteau measures combine both the specificity and sensitivity of the data by including measures of the probability that either the presence or absence of a particular land cover class is correctly classified. However, the remote sensing community are probably more familiar with the concepts of overall, user and producer accuracies and the methods suggested here may better reflect their needs.

This analysis used data collected as part of a standard remote sensing validation exercise. Whilst there is an extensive body of research describing the key issues in relation to validation data - see for example Stehman, (2000), Stehman et al. (2003), Stehman et al. (2005) and Stehman, (2009) - this is not the subject of this letter. Geographically weighted approaches compute a local statistic from data points under the kernel. The critical considerations for their implementation and the effective 
operation of the kernel include having a spatially distributed sample of data points over which to develop geographically weighted analyses and selecting an appropriate bandwidth.

Determining the bandwidth or the number of data points to include in the local model under the kernel is a trade-off between working with a dataset that is too small to calibrate the model reliably, and one that is too big such that it averages out local effects. Thus the aim is to select a bandwidth to ensure that enough data points are used in the calibration to minimise the cross validation prediction error. The kernel bandwidth kernel can be specified as a fixed distance or to include a proportion of the data points as in this research. Additionally it can be selected automatically by leave-one-out cross validation, which identifies a bandwidth for a given geographically weighted analysis that optimises the ability of the model to predict each individual response variable when it is removed from the dataset. Investigating the effects of applying different bandwidths offers a heuristic way of assessing the sensitivity of the data and the model with regard to cross validation prediction errors. Further details and discussion on bandwidth selection can be found in Fotheringham et al. (2002).

As well as developing geographically weighted user's and producer's accuracies, this research illustrates how geographically weighted methods provide a generic framework: the 'geographically weighted' approach is one that uses a kernel, under which any statistic can be calculated. By allowing bandwidths to vary, by using spatially dependent weights and by computing the statistic under the kernel using the distance weighted data, geographically weighted approaches address one of the fundamental tenets of geographical analyses: they operate under the assumption that the process under investigation vary continuously over space. In so doing they explicitly address Waldo Tobler's $1^{\text {st }}$ law of geography: “... but near things are more related than distant things" (Tobler, 1970, p236).

\section{Acknowledgements}

The author would like to thank Prof Brunsdon for advice on the use of geographically weighted models and Abdulhakim Khmag for the use of data collected during his $\mathrm{PhD}$. All of the statistical analysis and mapping were implemented in R version 2.15.1, the open source statistical software http://cran.r-project.org. The data and code used in this analysis will be provided to interested researchers on request.

\section{References}

BRUNSDON, C.F., FOTHERINGHAM, A.S. and CHARLTON, M., 1996, Geographically Weighted Regression - A Method for Exploring Spatial NonStationarity, Geographical Analysis, 28, pp. 281-298.

CAMPBELL, J., 1981, Spatial correlation effects upon accuracy of supervised classification of land cover. Photogrammetric Engineering of Remote Sensing, 47, pp. 355-364.

CANTERS, F., 1997, Evaluating the uncertainty of area estimates derived from fuzzy land-cover classification. Photogrammetric Engineering and Remote Sensing, 63, pp. 403-414. 
CHEN D.M. and WEI, H., 2009, The effect of spatial autocorrelation and class proportion on the accuracy measures from different sampling designs. ISPRS Journal of Photogrammetry and Remote Sensing, 64, pp. 140-150.

COMBER, A., FISHER, P.F., BRUNSDON, C. AND KHMAG, A., (in press), Spatial analysis of remote sensing image classification accuracy. Paper accepted for publication Remote Sensing of Environment (September, 2012)

CONGALTON, R. G., 1988, Using spatial auto-correlation analysis to explore the errors in maps generated from remotely sensed data. Photogrammetric Engineering and Remote Sensing, 54, pp. 587-592.

FOODY, G.M., 2002, Status of land cover classification accuracy assessment. Remote Sensing of Environment, 80, pp. 185-201.

FOODY, G. M., 2005, Local characterization of thematic classification accuracy through spatially constrained confusion matrices, International Journal of Remote Sensing, 26, pp. 1217-1228.

FOTHERINGHAM, A.S., BRUNSDON, C. and CHARLTON, M.E., 2002, Geographically Weighted Regression: The Analysis of Spatially Varying Relationships. Chichester: Wiley.

FOODY, G.M., 2007, Map comparison in GIS. Progress in Physical Geography 2007; 31, pp. 439- 445

FRIEDL, A. F., MCIVER, D. K., HODGES, J. C. F., ZHANG, X. Y., MUCHONEY, D.,STRAHLER, A. H., WOODCOCK, C. E., GOPAL, S., SCHNEIDER, A., COOPER, A., BACCINI, A., GAO, F. and SCHAAF, C., 2002. Global land cover mapping from MODIS: algorithms and early results. Remote Sensing of Environment, 83, pp. 287-302.

GONZALEZ, P., ASNER, G.P., BATTLES, J.J., LEFSKY, M.A., WARING, K.M. and PALACE, M., 2010, Forest carbon densities and uncertainties from Lidar, QuickBird, and field measurements in California, Remote Sensing of Environment 114, pp. 1561-1575.

LABOVITZ, M.L., 1984, The influence of autocorrelation in signature extraction - an example form a geobotanical investigation of Cotter basin, Montana. Photogrammetric Engineering and Remote Sensing, 52, pp. 201-211.

MCGWIRE, K. C., AND FISHER, P., 2001. Spatially Variable Thematic Accuracy: Beyond the Confusion Matrix. In C. T. Hunsaker, M. F. Goodchild, M. A. Friedl, \& T. J. Case (Eds.), Spatial Uncertainty in Ecology: Implications for Remote Sensing and GIS Applications (pp. 308-329). New York: SpringerVerlag.

STEELE, B.M., WINNE, J.C. and REDMOND, R.L., 1998, Estimation and mapping of misclassification probabilities for thematic land cover maps. Remote Sensing of Environment, 66, pp. 192-202.

STEHMAN, S.V., 2000, Practical implications of design-based sampling inference for thematic map accuracy assessment. Remote Sensing of Environment, 72, pp. $35-45$.

STEHMAN, S.V., SOHL, T.L., and LOEVELAND, T.R., 2003, Statistical sampling to characterize recent United States land-cover change. Remote Sensing of Environment, 86, pp. 517-529.

STEHMAN, S.V., SOHL, T.L., and LOVELAND, T.R., 2005, An evaluation of sampling strategies to improve precision of estimates of gross change in land 
use and land cover. International Journal of Remote Sensing, 26, pp. 49414957.

STEHMAN, S. V., 2009, Sampling designs for accuracy assessment of land cover. International Journal of Remote Sensing, 30 pp. 5243-5272.

TOBLER, W.R., 1970, A computer movie simulating urban growth in the Detroit region. Economic Geography 46, pp. 234-240. 


\section{Tables and Figures}

Table 1. The confusion matrix comparing reference and classified land cover classes, showing $60 \%$ overall agreement.

\begin{tabular}{|c|c|c|c|c|c|c|c|}
\hline \multirow{3}{*}{$\begin{array}{l}\text { Classified } \\
\text { Bare }\end{array}$} & \multicolumn{5}{|c|}{ Reference } & \multirow[b]{2}{*}{ Total } & \multirow[b]{2}{*}{ User } \\
\hline & Bare & Grazing Land & Urban & Vegetation & Woodland & & \\
\hline & 18 & 8 & 7 & 2 & 4 & 39 & 0.46 \\
\hline Grazing Land & 3 & 23 & 3 & 8 & 6 & 43 & 0.53 \\
\hline Urban & 0 & 0 & 27 & 1 & 2 & 30 & 0.90 \\
\hline Vegetation & 0 & 4 & 7 & 31 & 5 & 47 & 0.66 \\
\hline Woodland & 0 & 4 & 2 & 18 & 27 & 51 & 0.53 \\
\hline Total & 21 & 39 & 46 & 60 & 44 & 210 & \\
\hline Producer & 0.86 & 0.59 & 0.59 & 0.52 & 0.61 & & \\
\hline
\end{tabular}

Table 2. A summary of the variation in geographically weighted overall accuracy and Grazing Land user and producer accuracies.

\begin{tabular}{ccccccc} 
Accuracy & Min. & 1st Quartile & Median & Mean & 3rd Quartile & Max. \\
\hline Overall & 0.572 & 0.596 & 0.606 & 0.605 & 0.610 & 0.645 \\
User & 0.134 & 0.479 & 0.575 & 0.535 & 0.621 & 0.709 \\
Producer & 0.520 & 0.573 & 0.608 & 0.608 & 0.639 & 0.720 \\
\hline
\end{tabular}




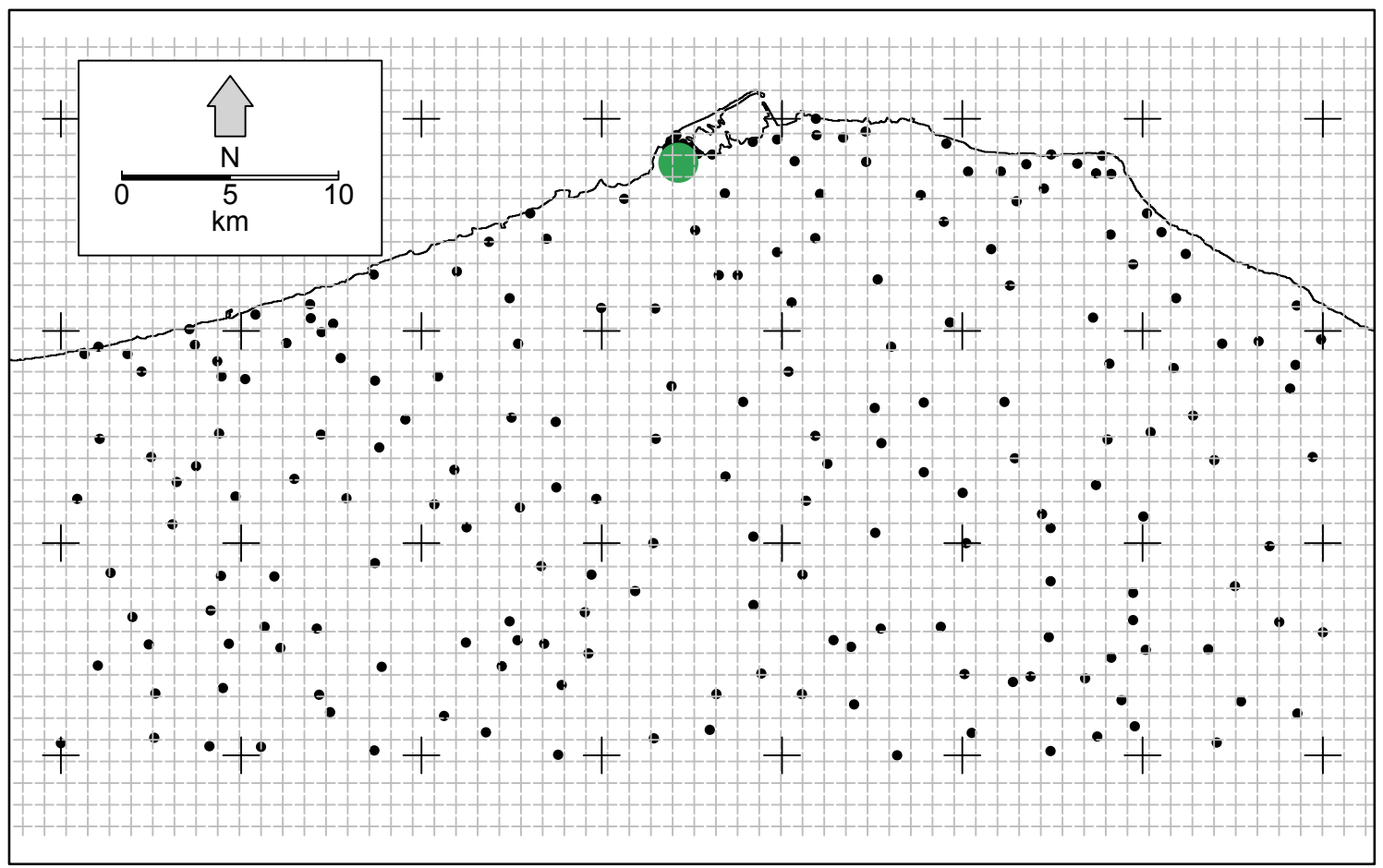

Figure 1 . The study area in Libya with the validation locations $(\bullet)$, the Tripoli area (shaded), the $1 \mathrm{~km}$ grid in grey over which the kernel was passed and the larger grid used to stratified the random sample of validation locations. 


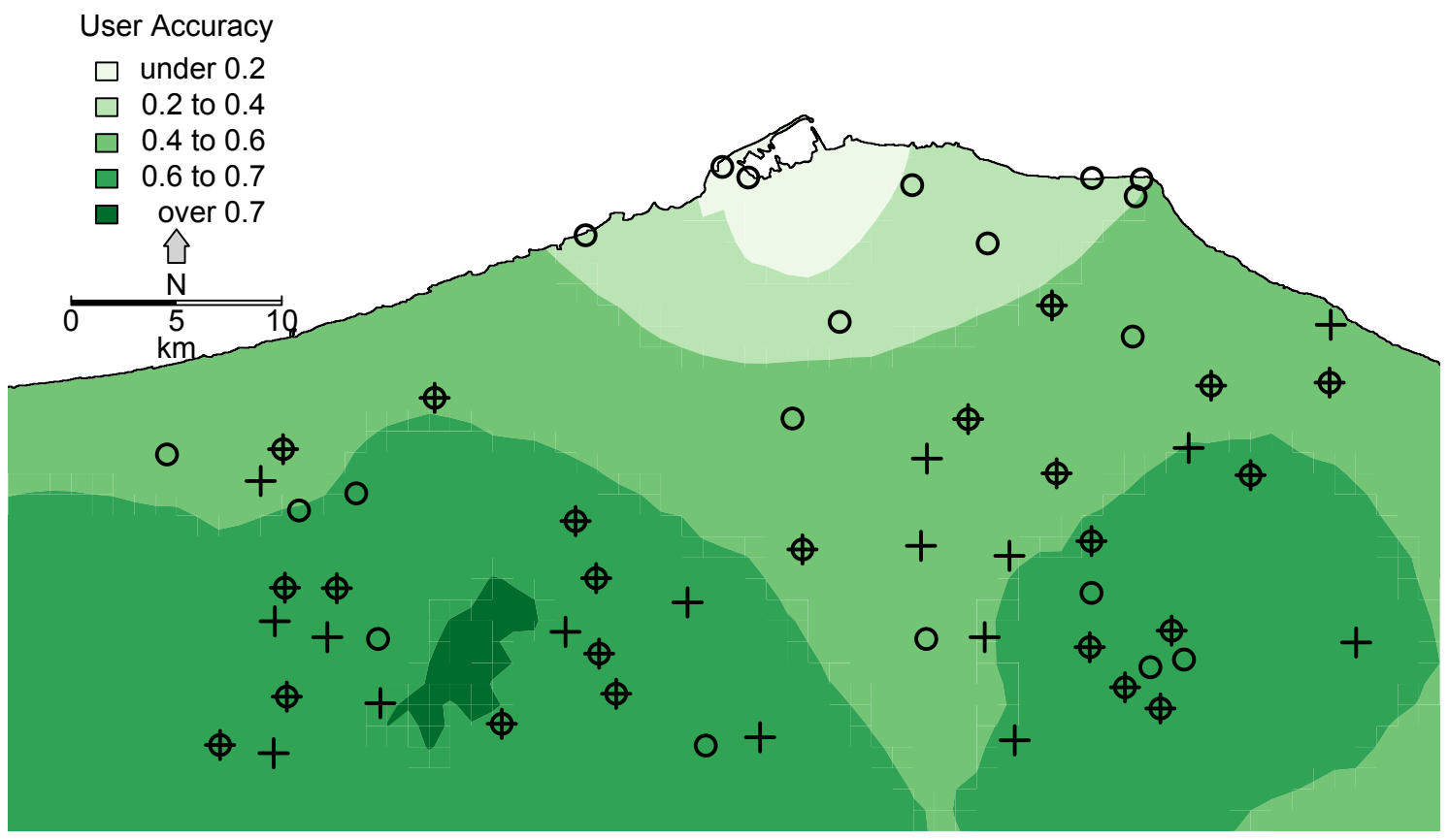

Figure 2. Spatial variation in user accuracy for the class of Grazing Land. Crosses indicate where Grazing Land was recorded in the reference data and circles show where it was recorded in the classified data. 


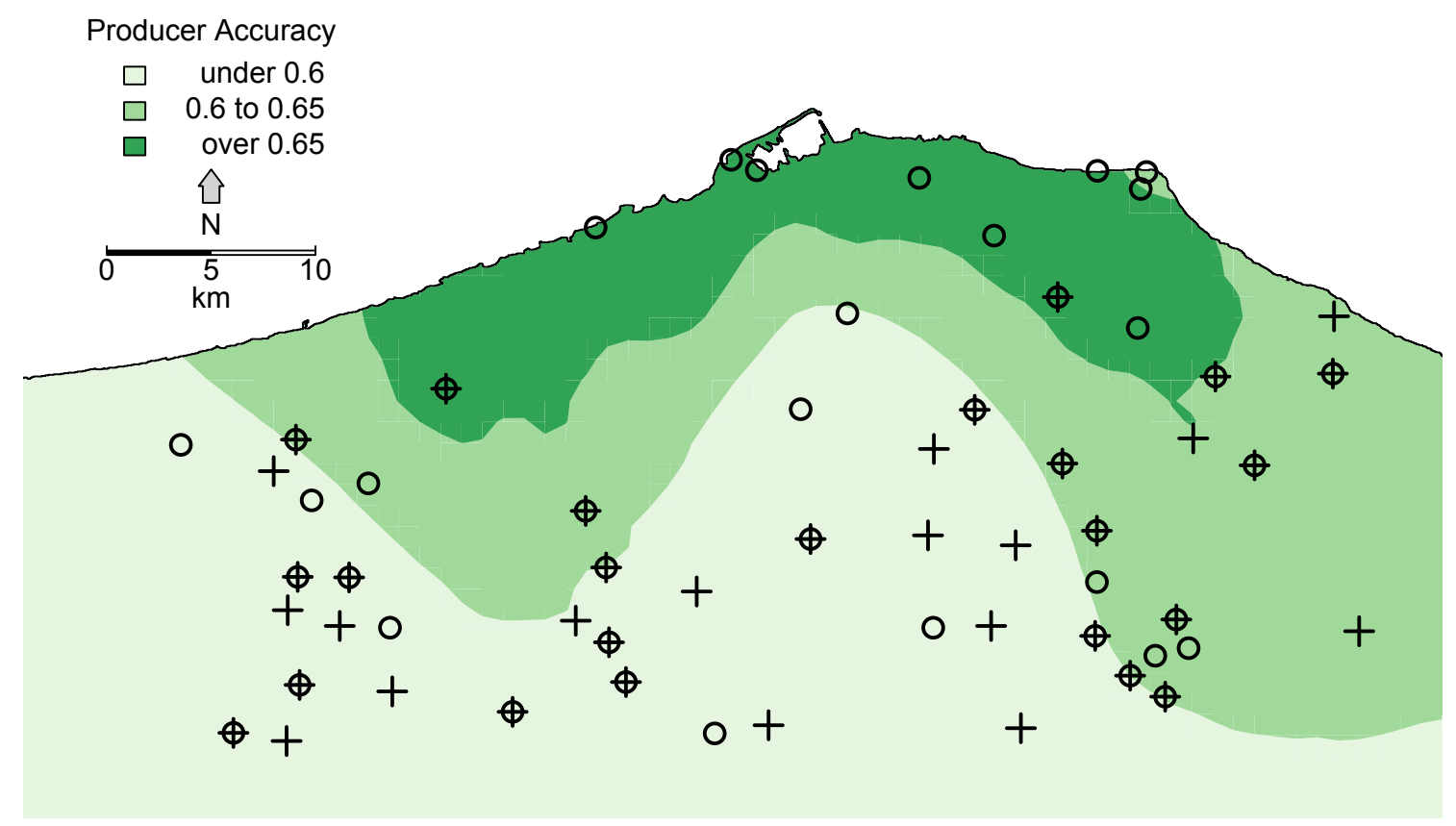

Figure 3. Spatial variation in producer accuracy for the class of Grazing Land. Crosses indicate where Grazing Land was recorded in the reference data and circles show where it was recorded in the classified data. 